\title{
Filigrane
}

Écoutes psychanalytiques

\section{L'enfant violent : quelques considérations pédopsychiatriques}

\section{Sylvaine De Plaen}

Volume 26, numéro 1, 2017

La terreur des enfants : première partie

URI : https://id.erudit.org/iderudit/1041690ar

DOI : https://doi.org/10.7202/1041690ar

Aller au sommaire du numéro

Éditeur(s)

Revue Santé mentale au Québec

ISSN

1192-1412 (imprimé)

1911-4656 (numérique)

Découvrir la revue

Citer cet article

De Plaen, S. (2017). L'enfant violent : quelques considérations

pédopsychiatriques. Filigrane, 26(1), 41-54. https://doi.org/10.7202/1041690ar

\section{Résumé de l'article}

Les problèmes de comportement et d'agressivité constituent une des raisons principales de consultation en pédopsychiatrie. Dans certaines situations, la violence et l'agressivité présentes peuvent devenir alarmantes et il devient alors nécessaire, au-delà de la nécessité d'agir et de contenir, de trouver des façons de continuer à penser et à réfléchir. Pour soutenir cette réflexion sur la violence, nous reviendrons tout d'abord sur les données issues de la recherche en paléoanthropologie sur l'évolution humaine et la place de l'agressivité dans le développement de la condition humaine. Également, les concepts développés par D. W. Winnicott, Philippe Jeammet, pédopsychiatre et psychanalyste, ainsi que Marie-Rose Moro, pédopsychiatre spécialisée en psychiatrie transculturelle et psychanalyste, contribueront également à nous éclairer sur les enjeux présents dans la clinique de la violence et de l'agressivité. Quatre vignettes cliniques sont également présentées pour illustrer cette complexité clinique.
Ce document est protégé par la loi sur le droit d'auteur. L'utilisation des services d’Érudit (y compris la reproduction) est assujettie à sa politique d'utilisation que vous pouvez consulter en ligne.

https://apropos.erudit.org/fr/usagers/politique-dutilisation/ 


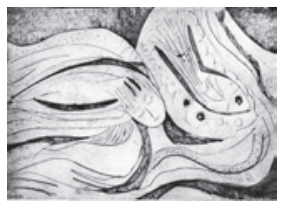

\title{
L'enfant violent: quelques considérations pédopsychiatriques
}

\author{
Sylvaine De Plaen
}

RÉSUMÉ: Les problèmes de comportement et d'agressivité constituent une des raisons principales de consultation en pédopsychiatrie. Dans certaines situations, la violence et l'agressivité présentes peuvent devenir alarmantes et il devient alors nécessaire, au-delà de la nécessité d'agir et de contenir, de trouver des façons de continuer à penser et à réfléchir. Pour soutenir cette réflexion sur la violence, nous reviendrons tout d'abord sur les données issues de la recherche en paléoanthropologie sur l'évolution humaine et la place de l'agressivité dans le développement de la condition humaine. Également, les concepts développés par D. W. Winnicott, Philippe Jeammet, pédopsychiatre et psychanalyste, ainsi que Marie-Rose Moro, pédopsychiatre spécialisée en psychiatrie transculturelle et psychanalyste, contribueront également à nous éclairer sur les enjeux présents dans la clinique de la violence et de l'agressivité. Quatre vignettes cliniques sont également présentées pour illustrer cette complexité clinique.

Mots clés: Violence, évolution humaine, pédopsychiatrie, psychanalyse, clinique.

\begin{abstract}
Agressive and problematic behaviours are the main causes for referral to child and adolescent psychiatric care. In some instances, the level of violence can become alarming and requires, beyond immediate containment, the ability to maintain avenues of thought and reflexion. For the purposes of such an endeavour, we will rely first on paleanthropological research findings on the evolution of humanity regarding agression. We will then refer to the contributions of Donald W. Winnicott, pediatrist and psychoanalyst, Philippe Jeammet, child psychiatrist and psychoanalyst, and Marie-Rose Moro from the field of transcultural child psychiatry and psychoanalysis, on the topic of violence and agression. Clinical vignettes will illustrate some of this issue's complexities.
\end{abstract}

Key words: violence, human evolution, child psychiatry, psychoanalysis

es problèmes de comportement et d'agressivité constituent certainement une des raisons principales de consultation en pédopsychiatrie. Dans certaines situations, la violence et la désorganisation présentes peuvent devenir alarmantes et commandent alors des interventions rapides pour contenir à la fois les frayeurs présentes et les risques réels de dérapage. Ces jeunes dont 
la violence inquiète au plus haut point l'entourage passent souvent en un premier temps par l'urgence pédopsychiatrique avant de se retrouver en clinique externe; dans un bon nombre de situations, ils sont cependant directement acheminés pour évaluation en clinique externe et sont alors priorisés au triage. Contenir, réfléchir et agir doivent alors souvent aller de pair et constituent de réels défis afin de ne pas succomber à la terreur et d'essayer de faire du sens. L'équipe multidisciplinaire devient alors un élément essentiel pour permettre de prendre du recul et gérer ces situations qui ont tendance à réveiller nos propres angoisses tout en anesthésiant notre pensée.

Pour introduire cette réflexion sur la violence et l'agressivité, je reviendrai tout d'abord sur des travaux récents effectués en anthropologie et en paléontologie autour de l'évolution humaine. Ces recherches riches et étonnantes constituent en effet une toile de fond privilégiée pour situer les enjeux liés à la violence dans un contexte socioculturel et historique beaucoup plus large. Nous reviendrons ensuite sur des vignettes évoquant chacune à leur façon de quelles manières les enjeux liés à la violence et à l'agressivité peuvent venir faire irruption dans notre espace clinique. Nous poursuivrons ensuite cette réflexion par un retour sur la pensée et les concepts développés par divers auteurs centraux dans le champ pédopsychiatrique autour de ces enjeux.

\section{Les racines de notre humanité}

Selon les scientifiques, l'espèce humaine qui est la nôtre, Homo sapiens, est apparue sur la terre africaine il y a environ 200000 ans, durant une période appelée le Paléolithique, ou première période de la préhistoire.

Bien avant, il y a environ 7 millions d'années, les lignages des ancêtres des humains et ceux des singes s'étaient déjà séparés; nous avons cependant conservé plus de $99 \%$ de notre matériel génétique commun avec les grands singes, comme le chimpanzé ou le gorille. Les premiers hominidés, nos ancêtres humains les plus anciens, étaient apparus en Afrique deux millions d'années avant l'apparition d'Homo Sapiens. La célèbre Lucy, autrefois considérée la grand-mère de notre humanité, n'en faisait pas directement partie; sa famille, celle des Australopithecus afarensis, a cependant persisté sur terre durant près de 900000 ans, pour s'éteindre il y a près de 3 millions d'années. C'est par la suite que vont apparaître deux nouvelles familles d'individus, dont le groupe des Paranthropus puis celui des Homo, nos lointains ancêtres, il y a 2,6 millions d'années.

$\mathrm{Au}$ cours de la longue histoire de la construction de notre humanité, deux grandes périodes de migration des premiers humains hors de l'Afrique 
ont eu lieu: une première grande vague de migration s'est produite autour de 250000 ans avant notre ère et s'est orientée d'abord vers le Proche-Orient puis vers l'Asie et vers l'Europe. C'est l'époque du règne de l'Homme de Néandertal sur toute l'Europe et une bonne partie du Moyen-Orient et de l'Asie, soit entre 250000 et 45000 ans. Une deuxième grande vague migratoire a eu lieu hors de l'Afrique autour de 50000 ans avant Jésus-Christ; c'est celle d'Homo sapiens, notre ancêtre direct. Cette nouvelle migration s'est d'abord dirigée vers le Moyen-Orient et ensuite vers l'Europe. Les découvertes archéologiques récentes ont mis en évidence le fait que ces deux espèces humaines, Homo sapiens et Homo neanderthalensis, ont bel et bien coexisté pendant près de 5400 ans dans certaines régions du monde, notamment en Europe.

Dans l'arbre généalogique bourgeonnant de nos ancêtres, on note également qu'au moins 5 espèces d'hominidés ont foulé la terre ferme en même temps vers 40000 ans avant notre ère. En plus d'Homo sapiens et de l'Homme de Néandertal, on peut aussi citer Homo erectus, l'Homme de Florès et enfin Homo naledi, nouvelle espèce la plus récemment identifiée en 2013, en Afrique du Sud. De toutes ces espèces, une seule a survécu, la nôtre. Si un certain métissage a pu être mis en évidence au niveau de l'ADN entre Homo sapiens et l'Homme de Néandertal (1 à $2 \%$ de l'ADN de l'homme moderne proviendrait de celle de Néandertal), une compétition semble également avoir pris place entre ces deux espèces, à la fois pour les ressources alimentaires et pour l'occupation du territoire. Cette compétition s'est notamment exprimée au niveau des naissances présentes de part et d'autre, des tueries entre groupes et également des meurtres entre individus.

Au-delà des affrontements et de l'agressivité exprimée à l'endroit des compétiteurs, une autre hypothèse est retenue pour expliquer la prédominance de nos ancêtres sur les autres espèces humaines; elle concerne la flexibilité particulière caractéristique d'Homo sapiens. Cette grande flexibilité, qui est présente par exemple au niveau du type d'alimentation ainsi que des capacités d'adaptation à des habitats divers, lui aurait permis de s'adapter à un environnement naturel alors en plein bouleversement. En effet, le climat aurait été marqué à cette période par une tendance globale à un environnement plus frais et plus sec, avec des oscillations régulières et rapides, nécessitant des adaptations rapides des êtres vivants. Dans un tel contexte, la capacité d'adaptation exceptionnelle de nos ancêtres leur aurait donné un avantage évolutif déterminant. En plus de la flexibilité présente au niveau des comportements et des pratiques, une autre caractéristique fondamentale de notre espèce semble être sa capacité à développer un intérêt pour l'état mental et les pensées d'autrui; cette 
capacité a notamment permis le développement d'une conscience des valeurs partagées par le groupe et a mené à la mise en place de normes sociales et de rôles spécifiques pour chacun. Cette capacité à s'organiser selon des normes, des valeurs et des objectifs communs a constitué un autre avantage évolutif déterminant pour nos ancêtres. Dans son numéro d'automne 2016, la revue Scientific American fait le point de façon fascinante sur l'état des connaissances actuelles concernant l'évolution de notre humanité.

\section{Les racines de la violence}

Des recherches récentes en anthropologie et en paléontologie mettent également de l'avant un héritage phylogénétique bien particulier pour l'homme moderne, soit une gestion particulière de la violence et de l'agressivité, notamment celle dirigée contre des membres de sa propre espèce. En effet, l'être humain semble avoir hérité de ses cousins les singes une certaine propension à la violence meurtrière entre congénères; cette violence serait même plus grande que celle de la moyenne des mammiferes. Le chercheur Jose Maria Gomez ainsi que ses collègues des Universités de Grenade et de Roi Juan Carlos de Madrid se sont penchés sur cet héritage phylogénétique de violence chez les animaux en général, et plus particulièrement chez les primates et les êtres humains. Ils se sont ainsi attachés à construire des modèles statistiques complexes tenant compte des liens de parenté entre espèces, en particulier celles appartenant aux mammifères, pour estimer les taux de violence meurtrière présents chez les premiers représentants de notre espèce. Ils ont notamment examiné la proportion de décès attribuables à la violence infligée par les congénères chez 1024 espèces de mammifères, ainsi que 600 populations humaines différentes et de différentes époques, allant du Paléolithique à nos jours. Ils ont ainsi démontré que si la violence meurtrière est rarissime, voire inexistante, chez les chauves-souris, les baleines et les lagomorphes (ordre qui comprend les lièvres et les lapins), elle est particulièrement fréquente chez les primates. Un autre élément qui participe des taux élevés de violence envers les congénères semble être l'importance de la territorialité pour les individus. Ainsi, il semble que la violence meurtrière soit plus élevée chez les espèces sociales et territoriales, comme le chimpanzé, par rapport à des espèces plus solitaires et peu attachées à un territoire, comme les grands singes Bonobos.

En tenant compte du caractère social et territorial de l'être humain et en incluant des informations sur son proche parent l'Homme de Néandertal, les chercheurs ont estimé que la violence intraspécifique chez Homo Sapiens constituait $2 \%$ des décès totaux, soit plus que 6 fois celle estimée pour les 
premiers mammifères. Le comportement social et la territorialité, deux traits comportementaux présents chez Homo Sapiens et ses proches parents, semblent avoir contribué à ce haut taux de violence meurtrière héritée phylogénétiquement.

Ce taux a cependant considérablement varié au cours de l'histoire de l'humanité: il était notamment plus élevé durant les périodes historiques plus anciennes ( $15 \%$, voire $30 \%$ il y a plus de 500 ans), et il a considérablement décliné à partir des temps modernes et de l'époque contemporaine. La culture, et notamment l'organisation sociopolitique particulière des sociétés humaines, paraît avoir joué un rôle significatif pour moduler cet héritage phylogénétique. Ainsi, les auteurs de la recherche soulignent que les stratégies mises en place par les sociétés pour contenir la violence, comme les services de police, les sanctions, les prisons et le rejet de la violence ont contribué à la baisse du taux de décès par homicide à $0,01 \%$ dans les sociétés modernes. On pourrait ajouter également que les valeurs transmises par l'éducation, tant au niveau familial qu'au niveau de l'école et de la communauté dans son ensemble, jouent également un rôle primordial pour contenir et canaliser cette violence potentielle nichée au cœur de l'humain.

La persistance de la violence envers les congénères au cours de l'évolution humaine semble ainsi avoir constitué une des stratégies fondamentales développées par notre espèce pour s'imposer, se protéger et se réguler en tant que groupe dans le monde souvent hostile et exigeant où elle a évolué. L'espèce humaine a par ailleurs également développé des stratégies pour contenir et gérer adéquatement cette même violence, stratégies tout aussi essentielles à notre survie en tant que groupe.

\section{La violence dans la clinique}

Les quatre vignettes suivantes évoquent chacune à leur façon comment la violence et l'agressivité peuvent venir faire intrusion, bousculer et parfois même bouleverser radicalement le travail en clinique pédopsychiatrique.

La première situation est celle de Johnny, un jeune garçon de 14 ans référé par son pédiatre pour de l'anxiété et des problèmes importants de comportement. Johnny ainsi que sa plus jeune sœur sont tous deux hébergés chez une tante maternelle, qui en a obtenu la garde légale deux ans plus tôt. La famille est originaire du Sud-Est asiatique. Suite à la séparation parentale survenue quand Johnny était en bas âge, Johnny et sa sœur ont vécu alternativement chez la mère et chez le père, qui se trouvaient tous deux incapables de s'en occuper. La mère avait notamment des problèmes de santé mentale 
sévères qui la rendaient constamment alitée et non disponible pour ses enfants. De son côté, le père était remarié, mais sa nouvelle conjointe avait une relation très conflictuelle avec les deux enfants. Depuis leur tout jeune âge, Johnny et sa sœur avaient vécu de la négligence, des abus physiques ainsi que des changements de milieux de vie répétés.

À son arrivée chez sa tante, Johnny était un enfant agité et qui faisait des crises de colère intenses au cours desquelles il pouvait devenir agressif physiquement et s'automutiler en se frappant la tête contre les murs. Il était également fréquemment opposant et n'avait aucune autonomie. Si les crises de colère avaient quelque peu diminué avec le temps, l'opposition et le manque d'autonomie avaient bel et bien persisté. Quand Johnny devenait plus anxieux ou plus préoccupé, il pouvait aussi se gratter la peau jusqu'au sang ou se ronger les ongles jusqu'à s'en faire des lésions au bout des doigts. Il mangeait très peu par ailleurs. En entrevue, avec sa taille frêle et menue, il ressemblait plutôt à un enfant de 8 ans. Au-delà des crises de colère et de l'opposition, la tante s'inquiétait également des mensonges et des petits vols fréquents de Johnny auprès de ses proches.

La deuxième situation concerne un enfant de 12 ans, Matthieu, référé suite à un geste suicidaire: dans un conflit avec d'autres jeunes à l'école, il avait voulu s'étrangler avec le cordon de son chandail. Matthieu était venu déjà trois fois à l'urgence pédopsychiatrique avant d'aboutir en clinique externe, dont la dernière fois deux semaines avant mon évaluation. Les raisons de consultation étaient toujours les mêmes: des idées suicidaires et des idées homicidaires.

Lors de la première rencontre d'évaluation en clinique externe, Matthieu venait d'être placé en centre d'accueil suite à un signalement à la DPJ fait lors d'une de ses visites à l'urgence. On notait aussi à l'histoire que Matthieu avait déjà vécu de l'intimidation quand il était en maternelle, ceci autant en milieu scolaire que dans le quartier où il résidait alors avec sa famille. Cette situation avait mené à un déménagement de toute la famille, mais les difficultés de fonctionnement avaient persisté par la suite. Il faut souligner que plusieurs évaluations avaient déjà été faites en milieu scolaire avant que je ne rencontre Mathieu, mettant notamment de l'avant un trouble d'apprentissage sévère, un raisonnement perceptif au niveau frontière, des difficultés d'attention ainsi que de l'anxiété.

Les intervenants scolaires se montraient très inquiets pour ce jeune et avaient insisté à répétition pour qu'il consulte à l'urgence et reçoive des soins pédopsychiatriques. Des incidents inquiétants étaient en effet survenus à 
l'école les semaines précédentes. Ainsi, à quelques reprises, Matthieu avait frappé un autre élève de l'école, menaçant de le tuer; il avait aussi menacé de se tuer lui-même par la suite lors d'un de ces incidents. Peu après, il avait été surpris en possession d'un couteau à l'école, confessant qu'il avait l'intention de tuer deux jeunes filles qui l'avaient intimidé la veille. On note que Matthieu était issu d'une famille fragile, avec un grand frère qui avait de sévères troubles de comportement. La mère, une jeune femme souriante et avenante, originaire des Îles du Pacifique, paraissait plutôt dépassée par la situation; le père, un Québécois ayant vécu une bonne partie de sa vie dans les Maritimes, avait perdu sa mère décédée de sclérose en plaques quelques années après sa naissance. Il avait également été l'objet de violence physique de la part de son père quand il était plus jeune.

La troisième situation est celle de Martin, un jeune garçon de 9 ans, référé par son pédiatre pour des troubles du comportement et des bizarreries comportementales. Martin était l'aîné de 2 enfants et n'avait jamais connu son père biologique. Deux signalements à la DPJ avaient déjà été faits dans le passé. La première fois, alors qu'il était encore en maternelle, Martin avait menacé une éducatrice de lui arracher les paupières. Il n'y avait pas eu de suite à l'époque. Deux ans plus tôt, un nouveau signalement avait été fait lorsque son frère ainé avait tenté de l'étrangler dans un accès de colère. On notait également à l'histoire que Martin semble habité par des idées particulièrement noires et morbides dès la garderie.

De leur côté, les intervenants scolaires rapportaient une escalade des comportements violents ainsi que des propos troublants depuis quelques mois. Ainsi, Martin pouvait dire qu'il avait des esprits qui le hantaient et qu'il devait prendre possession d'un groupe d'enfants de l'école afin de les recruter pour se défendre contre la mafia. Il était décrit comme souvent replié sur lui-même. Il pouvait aussi avoir des propos particulièrement violents envers les autres enfants, leur disant par exemple qu'il allait leur crever les yeux en les menaçant avec un trombone ou une paire de ciseaux. Il avait eu quelques fois des propos suicidaires. Ce fut le cas lors d'un incident où, jouant avec un objet non identifié dans le sable, il avait refusé de le montrer à l'éducateur du service de garde. Il avait alors menacé de se suicider avec le cordon de son chandail. En entrevue, Martin racontait que l'école n'avait pas le choix dans une telle situation: ou bien elle lui remettait l'objet en question, ou bien elle refusait et il mourait.

La dernière situation est celle d'un jeune de 10 ans, Joseph, référé à la fois par un médecin de la clinique des réfugiés ainsi que par ses intervenants 
scolaires pour trouble du comportement et agressivité. Joseph était arrivé au Québec avec ses deux parents et ses deux frères cadets en provenance de la Jordanie un an plus tôt avec un statut de réfugié. Les deux parents étaient d'origine arménienne, mais le père avait grandi en Syrie et la mère en Jordanie. La famille était chrétienne orthodoxe.

À l'histoire, il est apparu que Joseph avait été peu scolarisé avant son arrivée au Québec, avec au moins cinq changements d'école; cette situation avait fait suite aux déménagements nombreux de la famille pour des raisons diverses. La mère avait souvent trouvé son fils injustement ciblé et stigmatisé en milieu scolaire. Depuis son arrivée au Québec et son intégration en classe d'accueil, Joseph avait beaucoup de difficulté à suivre la routine de la classe ainsi que les exigences académiques. Il était décrit comme très rigide, réagissant à tout changement et tout imprévu par des crises de colère et des agressions physiques ciblées. Il était souvent menaçant envers les pairs et même les adultes suite à la moindre contrariété, ce qui fut le cas plus particulièrement avec un jeune musulman de sa classe venu du Moyen-Orient. À la maison, Joseph était aussi difficile et écoutait peu ses parents. Il pouvait quitter la maison sans avertissement et le père devait alors partir à sa recherche dans tout le quartier.

Ces quatre tableaux présentent des situations cliniques d'agressivité et de violence comportant à la fois certains points communs, mais également des caractéristiques distinctes. Afin d'être éclaircie un tant soit peu, chacune de ces situations a nécessité des investigations plus approfondies, le plus souvent en équipe multidisciplinaire, ainsi qu'un travail spécifique avec les partenaires du réseau, tant au niveau scolaire qu'au niveau de la première ligne en santé mentale. Décoder ce qui se trame derrière les comportements violents ou inhabituels de nos patients constitue souvent un réel défi, accentué par un fort sentiment d'urgence

\section{Quelques concepts théoriques autour de la violence et de l'agressivité}

Winnicott, pédiatre et psychanalyste anglais bien connu, est un des auteurs de référence les plus cités et les plus aimés en pédopsychiatrie. Sa formation à la fois de clinicien et de pédiatre a probablement contribué à la résonnance particulière de ses réflexions avec le travail clinique quotidien des pédopsychiatres. Un des éléments centraux du discours de Winnicott sur le développement de l'enfant est l'attention particulière qu'il accorde à l'environnement et au monde réel présent autour de l'enfant. En ce sens, 
un certain nombre de ses positions rejoignent celles développées par John Bowlby, lui-même pédopsychiatre et psychanalyste anglais, de la Clinique Tavistock à Londres, et connu comme le fondateur de la théorie de l'attachement. Les visions de Bowlby et Winnicott se rejoignent notamment en ce qui concerne leur vision de la délinquance et de la perte.

Dans ses travaux, Winnicott s'est particulièrement intéressé à la destructivité et à la tendance antisociale. Selon cet auteur, l'agressivité vient de l'intérieur, mais elle est aussi modulée par le dehors, en particulier par la qualité de la relation mère-enfant et de l'environnement précoce. Pour cet auteur, l'agressivité n'est pas perçue uniquement sous sa valence négative, mais aussi sous son aspect pulsionnel et moteur pour la vie. La tendance antisociale, comme la désigne Winnicott, est ici perçue comme un appel à l'autre et d'une certaine façon un signe de bonne santé mentale et d'espoir pour l'enfant. La pensée de Winnicott sur le thème de la destructivité et de la tendance antisociale chez l'enfant a été développée dans divers textes, textes qui ont été repris dans le recueil paru en 1984 chez Payot et intitulé Agressivité, culpabilité et réparation.

Ainsi, selon Winnicott, l'agressivité coexiste à l'origine avec l'amour chez l'enfant et tous deux se trouvent finement intriqués. L'agressivité pulsionnelle des débuts fait partie de l'appétit et d'une forme d'amour pulsionnel; elle s'accroît avec l'excitation et procure du plaisir à l'enfant. Winnicott fait aussi un lien entre cette agressivité précoce et le développement de la motricité chez l'enfant dès la période anténatale, avec les premiers mouvements et les heurts associés. Cette agressivité sous forme de motricité aidera progressivement l'enfant à différencier ce qui est le self et ce qui ne l'est pas. Les premiers coups de pied constituent ainsi le début de la relation avec les objets externes. Progressivement, le nourrisson se rend compte qu'il peut faire mal et il a envie d'aller de l'avant. En même temps, le désir de protéger l'être aimé amènera une inhibition des pulsions agressives présentes. Le rôle de la mère est ici primordial: en effet, si elle peut accompagner son bébé avec sensibilité, si elle peut être "suffisamment bonne», le nourrisson pourra renoncer à son contrôle magique sur le monde et à la tentation de la destruction. L'enfant s'autorisera suffisamment de satisfaction avec son agressivité pour ne pas être dangereux pour les personnes aimées. Pour cela, il a besoin de trouver à l'extérieur de lui quelqu'un qui pourra le frustrer mais aussi qui saura supporter sa haine. Il faudra que cet objet se montre capable de survivre aux attaques dont il est la cible de la part de l'enfant. Dans ce contexte, la pulsion destructrice de l'enfant se transformera éventuellement 
en désir de réparer, de construire et d'être responsable. Pour Winnicott, ce travail d'alchimie est à l'origine du jeu, du travail et de l'art.

Dans la pensée winnicottienne, l'enfant antisocial n'a pas pu se développer un bon environnement intérieur; il a absolument besoin d'être contrôlé par l'extérieur pour pouvoir se sentir heureux et être capable de travailler ou de jouer. L'enfant qui vole ne désire pas l'objet qu'il vole mais cherche plutôt quelque chose auquel il a droit: l'attention et l'amour de sa mère. Par ses actes, l'enfant recherche un environnement dont il a été privé dans sa tendre enfance, un lieu sûr, capable de lui imposer des limites, où ses impulsions et sa spontanéité seront en sécurité.

Winnicott parle également du double mouvement présent chez l'enfant qui lui permet d'édifier progressivement son monde interne: le fait de faire entrer quelque chose de l'extérieur (taking in) et de faire sortir quelque chose de lui (taking out), ceci autant sur le plan physique que psychique. Ces expériences sans cesse renouvelées d'absorption et d'expulsion vont permettre un remodelage continuel de son self et le développement de sa maturité affective.

À ce niveau, Winnicott rejoint les conceptions de Philippe Jeammet, pédopsychiatre, psychanalyste et professeur de psychiatrie de l'enfant et de l'adolescent à l'Institut mutualiste Montsouris à Paris. Dans son texte paru en janvier 2015 et intitulé La paradoxalité de l'être humain: dérégulateur des instincts et générateur de valeurs. Le paradigme de l'adolescence, Jeammet reprend cette idée d'échange obligatoire entre l'individu et son environnement. Il revient au mode de fonctionnement primordial de la cellule, qui rejette ce qui est mauvais et prend ce qui est bon, processus qui nécessite une membrane qui ne soit ni trop épaisse ni trop fragile. Pour Jeammet, il y a une tension permanente entre cette obligation de l'échange et le besoin d'individuation chez les êtres humains, en particulier chez les adolescents. Deux angoisses fondamentales sont issues de cette situation: d'une part, l'angoisse d'abandon et de ne pas être vu, de ne pas exister suffisamment pour les autres et, d'autre part, l'angoisse de fusion et d'intrusion, avec la peur de ne plus être maître de soi et de perdre ses limites.

Pour Jeammet, si l'on ne peut pas contrôler le vivant, on peut décider de le détruire. La destruction peut parfois être la seule chose qu'on maîtrise. De plus, elle permet de se libérer de la dépendance à l'autre et de se soulager psychologiquement. Pour cet auteur, la destructivité n'est pas simplement physique ou matérielle, elle peut aussi prendre le visage de toutes les conduites, attitudes ou croyances qui consistent à tenter d'effacer ou d'inverser les émotions qui peuvent nous rendre dépendants des autres. 
Comme Winnicott, Jeammet voit une appétence pour la vie derrière la destructivité. Les troubles de comportement peuvent avoir une valeur adaptative s'ils ne s'installent pas durablement et qu'ils n'entravent pas le développement de la personnalité. Le renforcement de ses assises narcissiques, c'est-à-dire la confiance en soi et en les autres, comme le sentiment d'avoir une valeur en soi et pour les autres, permettra à l'adolescent de recevoir ce dont il a besoin et de tolérer les relations auxquelles il aspire sans se sentir menacé. Pour Jeammet, on ne choisit pas d'aller mal ni de se faire du mal ou de prendre des risques excessifs, pas plus qu'on ne choisit de glisser sur une pente verglacée. Par contre, on peut être tenté de se laisser glisser, voire y prendre plaisir et penser que c'est notre choix. Il faudra permettre au sujet par la thérapie de retrouver progressivement une liberté de choix qui n'est possible que s'il acquiert une capacité minimale de prendre soin de lui et d'exister dans sa différence autrement qu'en s'attaquant lui-même.

En psychiatrie transculturelle, on a aussi considéré ce rapport particulier entre le dehors et le dedans pour le bon développement psychique, mais sous un angle un peu différent. Marie-Rose Moro, pédopsychiatre et psychanalyste, chef de file de la psychiatrie transculturelle française, insiste sur ce rapport particulier entre la culture du dedans, soit celle présente à l'intérieur de la famille, et celle du dehors, présente à l'extérieur de la famille, c'est-àdire dans la société d'accueil et à l'école. Les décalages vécus et expérimentés entre ces deux niveaux culturels peuvent contribuer de façon significative à diverses psychopathologies chez les jeunes, dont des troubles de comportement, du mutisme sélectif ou encore des difficultés d'apprentissage. Il faut aussi souligner que, parfois, les décalages culturels peuvent également exister au sein même des familles, par exemple en situation de couple mixte ou encore de jeunes dits de deuxième génération. Ces décalages deviennent source de souffrance quand ils provoquent un sentiment d'aliénation et de rupture dans celui de la continuité de soi, entravant à la fois le bon développement psychique de l'enfant et le bon fonctionnement de la famille. Par ailleurs, quand les enjeux autour des questions d'appartenance et de filiation ont été suffisamment négociés, la multiplicité des références devient alors source de métissage et de créativité.

Jeammet lui-même soulignait le rôle de la culture pour apporter des réponses organisées aux périodes de changement qui affectent les individus et les vulnérabilisent. Selon ces auteurs, l'étayage culturel semble avoir la capacité de prémunir l'enfant contre des ruptures possibles dans la construction et l'expérience de soi, le protégeant ainsi d'une décompensation ou 
d'une désorganisation éventuelles. Le bon fonctionnement culturel, avec son étayage protecteur et ses valeurs organisantes et structurantes pour l'identité, vient ainsi s'ajouter au bon fonctionnement de la famille pour aider l'enfant à faire face aux défis associés à son développement et à la gestion des liens entre son monde intérieur et le monde extérieur.

L'environnement culturel joue ainsi un rôle important pour soutenir le développement et l'organisation psychiques de l'enfant. Quand cet environnement plus large fait défaut, le bon développement des enfants et des adolescents devient à risque. Ceci peut être le cas dans diverses situations, par exemple suite à un processus d'immigration traumatique, à un conflit persistant au niveau des divers référents culturels de l'enfant ou encore parce que la société d'accueil devient elle-même génératrice de pratiques ou de discours violents ou aliénants. La stigmatisation et la discrimination génèrent une colère et une violence qui, loin d'être contenues par la société, peuvent aussi se trouver alimentées par les politiques en vigueur et les institutions sociales en place.

\section{Retour à la clinique}

Dans les cas cliniques présentés plus haut et dans la clinique de l'agressivité de façon plus générale, nous observons souvent une intrication complexe entre des facteurs de divers ordres venant altérer le bon développement des enfants et finissant par générer des comportements antisociaux ou violents. Parmi ces facteurs, la qualité de l'environnement familial et du lien parent-enfant apparaissent souvent constituer des facteurs majeurs dans le développement des comportements dysfonctionnels. Dans ces situations, il faut évaluer jusqu'où un travail thérapeutique sur les liens d'attachement ou encore un travail avec ou dans le milieu familial lui-même permettra de retrouver une stabilité et une sécurité suffisante pour que l'enfant puisse reprendre adéquatement son processus de développement. Ce travail doit souvent se faire en partenariat avec les autres réseaux de soin, comme les intervenants de première ligne ou encore les services sociaux. Parfois, retirer l'enfant d'un milieu familial trop toxique, trop négligent, ou juste incapable de se mobiliser, peut constituer l'étape déterminante de la prise en charge. Comme souligné plus haut, des sources de vulnérabilité peuvent également être présentes dans l'environnement plus large, comme l'école, la communauté ou la société, et être en lien avec des vécus traumatiques. Le travail thérapeutique doit alors en tenir compte et inclure la famille et d'autres membres du réseau, dont parfois des interprètes et des médiateurs culturels. 
Dans certains cas, des fragilités psychiques propres à l'enfant jouent un rôle de premier plan dans les difficultés présentées. Dans de telles situations, il peut y avoir une potentialisation explosive entre le fonctionnement psychique altéré de l'enfant et des fragilités du milieu familial qui n'arrive plus à répondre aux besoins et aux exigences de l'enfant. Évaluer la nature de ces fragilités par des investigations complémentaires, par exemple en psychologie, en neuropsychologie ou en orthophonie, peut alors devenir essentiel. Dans un certain nombre de cas, selon les vulnérabilités spécifiques mises en évidence, une médication peut être proposée pour procurer un soulagement à l'enfant, l'aider à mieux se réguler et également préserver ses liens sociaux mis à mal.

L'évaluation et la prise en charge des jeunes aux prises avec des comportements violents doivent donc tenir compte de cette multiplicité de facteurs. En conséquence, le travail pédopsychiatrique ne peut se déployer pleinement que dans un travail de partenariat et de complémentarité avec les autres professionnels des équipes pédopsychiatriques, comme les psychologues, travailleurs sociaux, orthophonistes et autres, mais également avec tous les intervenants du réseau, qu'il s'agisse de professionnels œuvrant au sein des CLSC, des écoles ou encore des services sociaux.

Comme êtres humains, nous avons hérité d'un lourd héritage phylogénétique qui favorise les comportements violents et l'agressivité, notamment envers les congénères. Nous savons aujourd'hui que nous sommes beaucoup plus proches de l'Homme de Néandertal que ce que nous pensions auparavant, et également que nous sommes encore très proches des grands singes, nos cousins. Au-delà de cet héritage de violence meurtrière, notre espèce humaine s'est aussi construite et définie autour de caractéristiques qui paraissent spécifiques aux humains et qui nous rendent distincts de nos cousins primates. Ces caractéristiques ont été notamment mises en évidence dans diverses expériences de laboratoire, notamment au Laboratoire de l'Institut Max Planck en Allemagne, qui a comparé les performances de jeunes enfants et de chimpanzés dans différentes tâches cognitives. L'attention conjointe, qui signe le désir de partager un intérêt ou une intention avec l'autre, est observée chez de jeunes enfants mais n'est pas observée chez les chimpanzés; elle constitue ainsi un des premiers signes de l'élaboration de la capacité de mentalisation au cours du développement de l'enfant. La mentalisation et les cognitions sociales semblent constituer des attributs humains spécifiques qui favorisent la sensibilité à l'autre, la coopération et la solidarité. En plus de soutenir un développement dynamique de l'identité, elles 
permettent l'inscription de l'individu dans un groupe d'appartenance ainsi qu'une complexification du réseau des liens sociaux. C'est en s'appuyant sur les caractéristiques spécifiquement humaines de la flexibilité, de la mentalisation et de la gestion des liens sociaux que la pédopsychiatrie tente d'adresser cliniquement les comportements dysfonctionnels associés à l'agressivité et à la violence.

Sylvaine De Plaen deplaen@videotron.ca

\section{Références}

De Menocal, P. B. (2016). Climate Shocks. Scientific American, 25 (4), 22-27.

Gomez, J. M., Verdu, M., Gonzalez-Megias, A. et Mendez, M. (2016). The phylogenetics roots of human lethal violence. Nature, 538, 233-237.

Gravel, P. (2016, 22 oct.). Réhabiliter l'Homme de Néandertal. Celui qu’on imagine comme un être mal dégrossi n'a rien à envier à Homo Sapiens. Le Devoir.

Jeammet, P. (2015). La paradoxalité de l'être humain: dérégulateur des instincts et générateur de valeurs. Le paradigme de l'adolescence. Dans Frydman, R., et Flys-Treve, M. (dir.). Mesure et démesure... Peut-on vivre sans limites? (p. 105-123). Paris: Presses universitaires de France.

Hammer, M. F. (2016). Human Hybrids. Scientific American, 25 (4), 44-49.

Pringle, H. (2016). The Origins of Creativity. Scientific American, 25 (4), 86-93.

Slix, G. (2016). The «It» Factor. Scientific American, 25 (4), 60-65.

Winnicott, D. W. (1984). Agressivité, culpabilité et réparation. Paris: Payot, 2016.

Wong, K. (2016). Neandertal Minds. Scientific American, 25 (4), 66-73.

Wood, B. (2016). Welcome to the Family. Scientific American, 25 (4), 6-11. 